\title{
The relation between left ventricular global longitudinal strain and troponin levels in patients hospitalized with COVID-19 pneumonia
}

\author{
Betul Balaban Kocas ${ }^{1} \oplus \cdot$ Gokhan Cetinkal $^{1} \cdot$ Ozgur Selim Ser $^{1} \cdot$ Hakan Kilci $^{1} \cdot$ Kudret Keskin $^{1} \cdot$ Safiye Nur Ozcan ${ }^{2}$. \\ Yildiz Verdi ${ }^{2} \cdot$ Mustafa Ismet Zeren ${ }^{2} \cdot K_{\text {Kadriye Kilickesmez }}{ }^{1}$
}

Received: 2 July 2020 / Accepted: 8 November 2020 / Published online: 18 November 2020

(c) Springer Nature B.V. 2020

\begin{abstract}
Left ventricular global longitudinal strain (LVGLS) from two-dimensional speckle-tracking echocardiography (2D-STE) provides a more accurate estimation of subclinical myocardial dysfunction. In patients with COVID-19, elevated high sensitive troponin (hs-TnI) levels are frequent independent from the underlying cardiovascular disease. However, the relationship between high troponin levels and LVGLS in such patients remains unknown. We aimed to investigate the relation between troponin levels and LVGLS values in patients with COVID-19. A total of thirty-eight patients diagnosed with COVID-19 pneumonia who underwent echocardiography examination within the first week of hospital admission were enrolled in our study. Patients were divided into two groups according to their hs-TnI levels. Conventional left venticular (LV) function parameters, including ejection fraction, LV diastolic and systolic volumes were obtained and LVGLS was determined using 2D-STE. Frequency of hypertension, diabetes mellitus and current smoking were similar among groups. Compared with the patients in the negative troponin group, those in the positive troponin group were more likely to have a higher age; higher levels of D-dimer, C-reactive protein and ferritin; higher need for high-flow oxygen, invasive mechanical ventilation therapy or both; and a higher number of intensive care unit admissions. There was no statistically significant difference in LVGLS and ejection fraction values between the two groups. $(-18.5 \pm 2.9,-19.8 \pm 2.8, \mathrm{p}=0.19 ; 55.2 \pm 9.9,59.5 \pm 5.9, \mathrm{p}=0.11$ respectively). Despite troponin increase is highly related to in-hospital adverse events; no relevance was found between troponin increase and LVGLS values of COVID-19 patients.
\end{abstract}

Keywords COVID-19 2 2D speckle tracking echocardiography $\cdot$ Left ventricular global longitudinal strain $\cdot$ High sensitive troponin

\section{Introduction}

In December 2019, a novel coronavirus (2019-nCoV) was initially identified in the respiratory tract cells of patients hospitalized with pneumonia in Wuhan, China. Coronavirus disease 2019 (COVID-19) caused by this new type of virus has emerged as a serious public health issue due to its rapid human-to-human transmission. Since then, it has become

Betul Balaban Kocas

betulbalaban@yahoo.com

1 Department of Cardiology, Sisli Hamidiye Etfal Education and Research Hospital, Sisli, Mecidiyekoy, 34360 Istanbul, Turkey

2 Department of Infectious Disease and Microbiology, Sisli Hamidiye Etfal Education and Research Hospital, Sisli, Istanbul, Turkey one of the most common causes of mortality and morbidity worldwide.

It has been reported that the 2019-nCoV may influence a large variety of organs besides the lungs and lead to multiple organ failure. Prior studies suggest that severe COVID-19 may present with acute cardiac involvement $[1,2]$ and myocardial injury is more likely related to secondary reasons rather than direct toxic effect of the virus [3]. Cardiac damage manifested mainly by high sensitive troponin-I (hs-TnI) elevation is a quite common finding among these patients and seems to be strongly related to adverse clinical outcomes [4]. These findings imply that, early identification of patients with myocardial injury may be vital to preventing poor outcomes and fatality [5].

Moreover, a myocardial injury may result in clinical or subclinical impairment of left ventricular (LV) systolic function in patients affected by COVID-19. Although LV 
ejection fraction (LVEF) is the most widely used measurement for evaluating LV systolic function in daily clinical practice, intra-observer and inter-observer variabilities and difficulty in endocardial border tracing are the main limitations. Global longitudinal strain (GLS) obtained by twodimensional speckle tracking echocardiography (2D-STE) is a recently used sensitive and validated method [6] for early detection of subclinical LV dysfunction according to the European and American Society of Echocardiography guidelines. It permits evaluation of the myocardial strain, thereby providing detailed data about the LV deformation. It has also been reported that LVGLS presents more objective information than LVEF [7] and provides additional prognostic information.

A recent study conducted in a large population with no prior cardiovascular disease showed that troponin increase was inversely associated with GLS values [8]. Therefore, we assumed that 2D-STE might detect subclinical LV dysfunction in hospitalized COVID-19 patients with elevated troponin levels. Due to the lack of data regarding the association between LVGLS and elevated levels of hs-TnI in patients with COVID-19 pneumonia; we aimed to investigate whether mild troponin increase caused by COVID-19 pneumonia itself, has a relation with LVGLS in these group of patients. Hence, comprehensive evaluation of this question may provide additional information about management of the patients with troponin increase during their hospitalization for COVID-19.

\section{Materials and methods}

A total of 128 consecutive adult patients who were diagnosed with COVID-19 from April 4, 2020 to May 7, 2020 were enrolled in our single-center, prospective and observational study. Except COVID-19, all other clinical conditions that may increase troponin levels or impair LVGLS analysis were determined as the exclusion criteria. In this respect, patients with older age ( $>85$ years old), preexisting cardiovascular disease, arrhythmia, $\geq$ stage 2 hypertension(HTN), uncontrolled diabetes mellitus(DM) (HbA1c $\geq 8)$, prior cerebrovascular disease, chronic liver or kidney disease (GFR $<30 \mathrm{ml} / \mathrm{min}$ ), hemodialysis, severe chronic obstructive pulmonary disease (COPD), malignancy and poor echogenicity were excluded. Of the screened patients, the following were excluded: 16 owing to older age ( $>85$ years old), 22 due to the presence of previous cardiovascular disease, eight due to arrhythmia and poor echogenicity, six due to chronic kidney disease or heamodialysis, 11 due to uncontrolled HTN or DM, three due to prior cerebrovascular disease, six due to severe COPD and 18 were due to frailty and oncologic problems. This resulted in 38 research subjects meeting the criteria for final analysis.(Fig. 1) Our naive

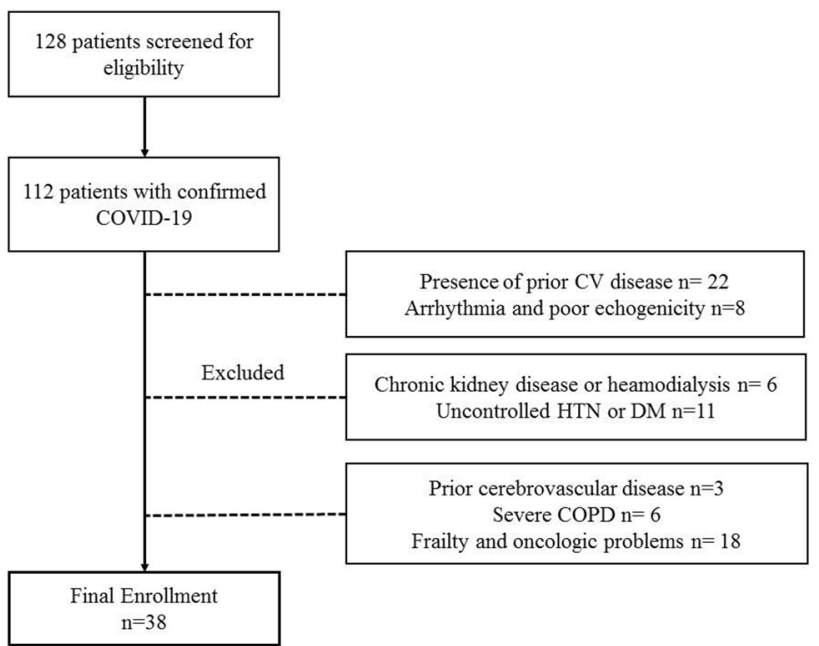

Fig. 1 Study flow chart. COVID-19 coronavirus disease 2019, CV cardiovascular, HTN hypertension, DM diabetes mellitus, COPD chronic obstructive pulmonary disease, $h s-T n I$ high sensitive troponin I

study population was categorized into two groups; group 1: 11 patients with elevated troponin levels and group 2: 27 patients with negative troponin levels. Besides, we wanted to demonstrate the effect of age on baseline echocardiographic characteristics and clinical endpoints of the study population. Study population was additionally divided into two groups according to age as; Group 1 age $\geq 65$ years $(n=11)$ and Group 2 age $<65$ years $(n=27)$ in Table 3 .

Patients with a serum level of hs-TnI above the 99th-percentile of the upper reference limit were considered as hs - TnI positive. The upper limit of the troponin test kit studied in our laboratory was $11.6 \mathrm{ng} / \mathrm{L}$. Inclusion criteria regarding the troponin level was arbitrarily set to up to 10 times above the 99th-percentile of upper reference limit since we aimed to investigate the effect of troponin increase on LV systolic function.This study was yielded with the edicts of the 1975 Declaration of Helsinki and was approved by the local Ethics Committee of University of Health Sciences Sisli Hamidiye Etfal Training and Research Hospital (No:2734/2020).

Demographic, laboratory and clinical information were gathered during hospitalization by associated physicians from electronic medical records. Demographic and clinical data included age, gender, body surface area (BSA), body mass index (BMI), presence of DM, HTN and length of hospital stay. Blood test results inclusive of complete blood count, renal and liver function tests, D-dimer, procalcitonin, $\mathrm{C}$ reactive protein(CRP), ferritin and hs-TnI were recorded. The laboratory data was collected on the day of echocardiogram procedure. In hospital adverse outcomes were defined as high-flow oxygen demand, invasive mechanical ventilation therapy (intubation) demand, or both, intensive care unit (ICU) admission. 
All patients underwent bedside echocardiographic examination within the first week of hospital admission using the same machine (EPIQ 7, Philips Medical Systems, Andover, MA, USA) with a 5-1 MHz sector transducer. Images were obtained by two trained cardiologists (BBK and GC) who were blinded for clinical trial data, following the recommendations of the American Society of Echocardiography [9] All echocardiographic data were analyzed offline with a commercially available software (QLab). Left heart diameters, thickness of the interventricular septum and the LV posterior wall were determined according to the recommendations of the American Society of Echocardiography guideline [9]. LV end-systolic volume(ESV), end-diastolic volume(EDV) and EF were measured using the modified Simpson's rule. Left atrial volume was calculated using the biplane method in four- and two-chamber views, and it was indexed for BSA. LV mass (LVM) was calculated using the Devereux's formula (0.8 \{1.04 [(LVIDD+ PWTD + IVSTD) (3) $-($ LVIDD)(3)] $\}+0.6 \mathrm{~g}$ ) and LVM was indexed by BSA. LV diastolic function was determined by the early transmitral flow velocity (E), to the late transmitral flow velocity (A): E/A ratio, and $\mathrm{E}$ wave deceleration time and the transmitral $\mathrm{E}$ to the mean of early diastolic LV septal and early diastolic LV lateral tissue velocities (mean e'): E/mean e' ratio.Right ventricular (RV) size was determined from the apical 4-chamber view. Tricuspid annular plane systolic excursion (TAPSE) was recorded on M-mode and tricuspid lateral annular systolic velocity ( $\left.\mathrm{S}^{\prime}\right)$ was assessed by tissue Doppler imaging from the apical 4 -chamber view.
Myocardial GLS was measured using the Philips EPIQ7 machine by using commercially available software (QLAb) for the LV. (Fig. 2) The mean GLS was calculated by averaging the peak GLS values of three apical views (2-, 3and 4-ch) and automatic endocardial contour tracking was performed in end-systole. The timing of end-systole was determined by aortic valve closure. LVGLS assessment was carried out by a single pyhsician (BBK). The normal limit of GLS, which is expressed as a negative value, was $<-18 \%[7,10]$. In other words, the more negative values demonstrate the better cardiac function.

Continuous variables were reported as means \pm standard deviation (SD), while categorical variables were presented as percentages. The normality tests for continuous variables were determined with the Kolmogorov-Smirnov test. The categorical variables between the groups were evaluated with the chi-square test. For continuous variables, the normally distributed data were evaluated with the Student's t-test and the data which was not normally distributed were evaluated with the Mann-Whitney U test. Multivariate logistic regression analysis was performed to evaluate the independent predictors of LVGLS $>-18$ and adverse clinical events. Values of $\mathrm{p}<0.05$ were considered statistically significant. SPSS 22 software (SPSS Inc, Chicago, Illinois) was used to perform all statistical analysis.

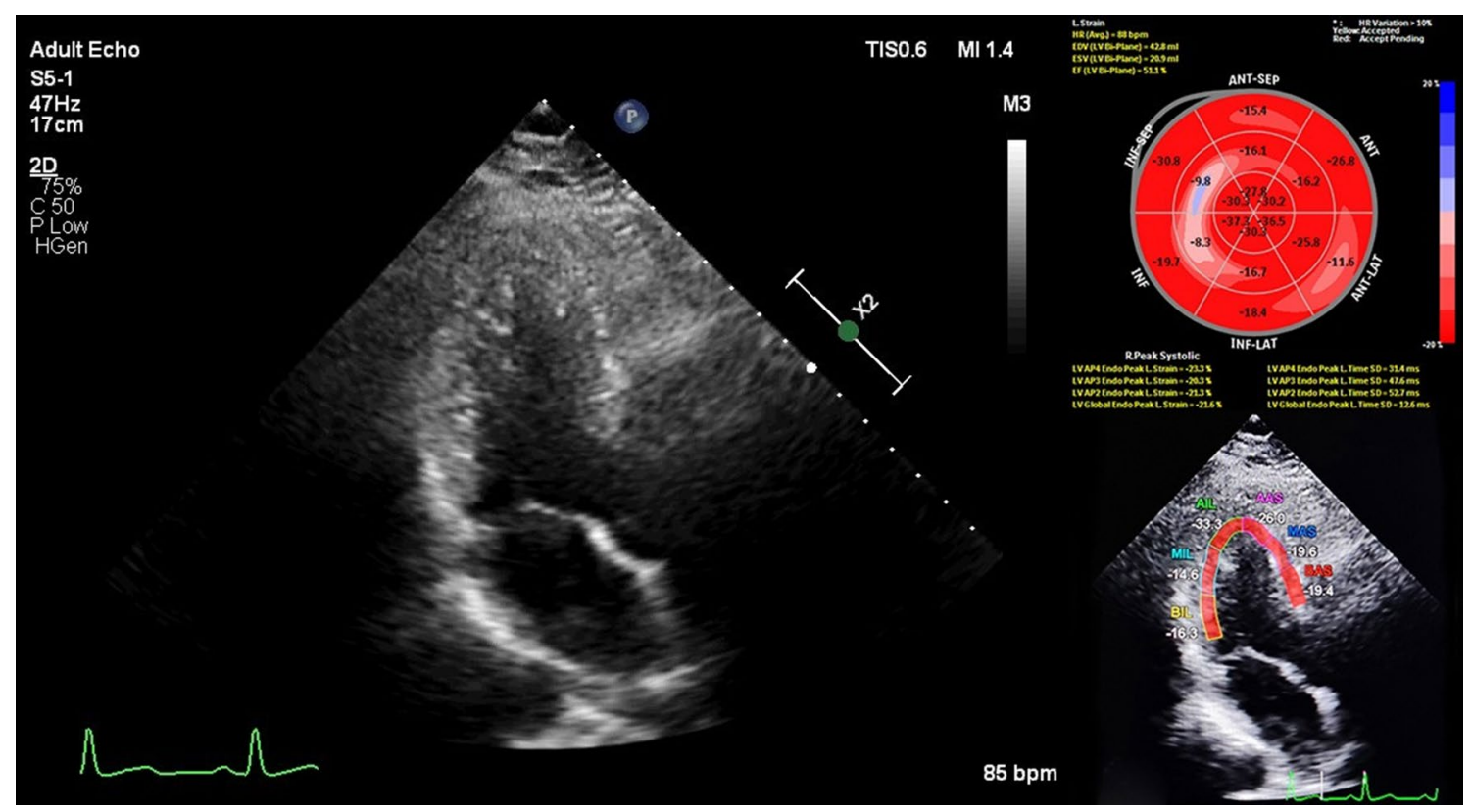

Fig. 2 Speckle tracking apical three chamber view showing global longitudinal strain 


\section{Results}

The demographic, clinical and laboratory data of the subjects were shown in Table 1 . There were 22 males $(57.9 \%)$ and 16 females $(42.1 \%)$. The mean age of the patients was $54.4 \pm 12.9$ years (minimum 20, maximum 81 ). The positive troponin group was significantly older than the negative troponin group. There was no statistically significant difference between the groups in terms of DM, HTN, sex, smoking rates and BMI levels. Medical treatment of COVID-19 pneumonia was similar between the groups except for oseltamivir and favipiravir therapy. Compared with patients in the low troponin group, those in the high troponin group were more likely to have a higher D-dimer, CRP, procalcitonin, ferritin, creatinine, alanine transaminase (ALT), aspartate transaminase (AST) and lactate dehydrogenase (LDH) levels. There was no statistically significant difference in glucose levels and complete blood count parameters between the two groups.

The echocardiographic characteristics of the study group were described in Table 2. Compared with patients in the low troponin group, those in the high troponin group had similar LVGLS and EF levels. Also, transmitral flow parameters and tissue Doppler parameters except septal e' wave velocity showed no difference. Patients in the positive troponin group exhibited more dilated left atrium and left ventricle, higher LVEDV, left atrial volume and volume index. The thickness of the interventricular septum and LV posterior wall, LVM and LVMI tended to be higher in the positive troponin group. At the same time, TAPSE, RV diameter, $S$ ' velocity and the number of patients with GLS $\geq-18$ did not differ between

Table 1 The demographic and clinical data of the study group according to hs-TnI levels

\begin{tabular}{|c|c|c|c|c|}
\hline & All patients $(\mathrm{n}=38)$ & Positive Hs TnI $(n=11)$ & Negative Hs TnI $(n=27)$ & $\mathrm{p}$ value \\
\hline Age (years) & $54.4 \pm 12.9$ & $66.3 \pm 10.4$ & $49.6 \pm 10.6$ & $<0.001^{\mathrm{a}}$ \\
\hline Male gender & $22(57.9 \%)$ & $8(72.7 \%)$ & $14(51.9 \%)$ & 0.23 \\
\hline $\operatorname{BSA}\left(\mathrm{m}^{2}\right)$ & $1.9 \pm 0.17$ & $1.94 \pm 0.14$ & $1.92 \pm 0.18$ & 0.74 \\
\hline BMI $\left(\mathrm{kg} / \mathrm{m}^{2}\right)$ & $28.2 \pm 3.9$ & $28.9 \pm 3.8$ & $27.9 \pm 3.9$ & 0.49 \\
\hline HTN & $13(34.2 \%)$ & $5(45.5 \%)$ & $8(29.6 \%)$ & 0.35 \\
\hline $\mathrm{DM}$ & $5(13.2 \%)$ & $2(18.2 \%)$ & $3(11.1 \%)$ & 0.56 \\
\hline Smoking & $5(13.2 \%)$ & $2(18.2 \%)$ & $3(11.1 \%)$ & 0.56 \\
\hline Length of hospital stay (days) & $14.2 \pm 9$ & $20.8 \pm 8.9$ & $11.4 \pm 7.7$ & 0.003 \\
\hline \multicolumn{5}{|l|}{ Medications } \\
\hline Hydroxychloroquine & $37(97.4 \%)$ & $11(100 \%)$ & $26(96.3 \%)$ & 0.99 \\
\hline Oseltamivir & $11(28.9 \%)$ & $6(54.5 \%)$ & $5(18.5 \%)$ & 0.03 \\
\hline Favipiravir & $13(34.2 \%)$ & $9(81.8 \%)$ & $4(14.8 \%)$ & $<0.001$ \\
\hline Azithromycine & $11(28.9 \%)$ & $3(27.3 \%)$ & $8(29.6 \%)$ & 0.88 \\
\hline Enoxaparin & $37(97.4 \%)$ & $11(100 \%)$ & $26(96.3 \%)$ & 0.99 \\
\hline $\mathrm{WBC}\left(/ \mathrm{mm}^{3}\right)$ & $7400 \pm 4200$ & $8233 \pm 3270$ & $7060 \pm 4535$ & 0.44 \\
\hline Hemoglobin (g/dL) & $13.6 \pm 1.7$ & $13.3 \pm 1.9$ & $13.7 \pm 1.7$ & 0.57 \\
\hline Neutrophil (/mm³) & $5390 \pm 2820$ & $6700 \pm 2947$ & $4862 \pm 2642$ & 0.06 \\
\hline Lymphocyte $\left(/ \mathrm{mm}^{3}\right)$ & $1258 \pm 575$ & $1121 \pm 530$ & $1314 \pm 593$ & 0.35 \\
\hline Platelet $\left(10^{3} / \mathrm{mm}^{3}\right)$ & $193.9 \pm 68$ & $190.5 \pm 75.7$ & $195.3 \pm 66$ & 0.84 \\
\hline Glucose (mg/dL) & $122.8 \pm 36.4$ & $135.1 \pm 38$ & $117.8 \pm 35.2$ & 0.18 \\
\hline Creatinine $(\mathrm{mg} / \mathrm{dL})$ & $0.90 \pm 0.27$ & $1.05 \pm 0.4$ & $0.84 \pm 0.17$ & 0.03 \\
\hline ALT (U/L) & $29.2 \pm 15.4$ & $35.3 \pm 15.9$ & $26.7 \pm 14.8$ & 0.12 \\
\hline AST (U/L) & $37.9 \pm 21.7$ & $53 \pm 29.8$ & $31.8 \pm 14$ & $0.007^{\mathrm{a}}$ \\
\hline hs-TnI (ng/L) & $23.9 \pm 36.3$ & $70.1 \pm 39$ & $5.1 \pm 4.2$ & $<0.001^{\mathrm{a}}$ \\
\hline D-dimer (ug/L) & $928.7 \pm 686.5$ & $1264.1 \pm 626.8$ & $791.9 \pm 672.7$ & $0.006^{\mathrm{a}}$ \\
\hline Ferritin (ug/L) & $305.9 \pm 302$ & $555 \pm 334.7$ & $204.5 \pm 223.3$ & $0.002^{\mathrm{a}}$ \\
\hline Procalcitonin (ug/L) & $0.23 \pm 0.18$ & $0.38 \pm 0.21$ & $0.17 \pm 0.13$ & $<0.001^{\mathrm{a}}$ \\
\hline CRP (mg/L) & $84.3 \pm 69.8$ & $157.4 \pm 55.9$ & $54.5 \pm 50.6$ & $<0.001^{\mathrm{a}}$ \\
\hline LDH (U/L) & $319.8 \pm 134.7$ & $434.6 \pm 134$ & $273.1 \pm 105.4$ & $<0.001^{\mathrm{a}}$ \\
\hline
\end{tabular}

hs-TnI high sensitive troponin-I, $B S A$ body surface area, $B M I$ body mass index, $H T N$ hypertension, $D M$ diabetes mellitus, $W B C$ white blood cell, $A L T$ alanine transaminase, $A S T$ aspartate transaminase, $C R P C$ reactive protein, $L D H$ lactate dehydrogenase

${ }^{a}$ Mann Whitney-U test 
Table 2 The echocardiographic variables of the study group according to hs-TnI levels

\begin{tabular}{|c|c|c|c|c|}
\hline & All patients $(\mathrm{n}=38)$ & Positive hs-TnI $(n=11)$ & Negative hs-TnI $(\mathrm{n}=27)$ & $\mathrm{p}$ value \\
\hline LVDd (mm) & $44.3 \pm 4.1$ & $47.2 \pm 4.9$ & $43.1 \pm 3.2$ & 0.004 \\
\hline IVSd (mm) & $10.1 \pm 1.5$ & $11.4 \pm 1.3$ & $9.6 \pm 1.2$ & $0.001^{\mathrm{a}}$ \\
\hline PWd (mm) & $9.9 \pm 1.3$ & $10.9 \pm 0.9$ & $9.5 \pm 1.2$ & $0.002^{\mathrm{a}}$ \\
\hline LAd (mm) & $35.6 \pm 4.7$ & $40.4 \pm 4.3$ & $33.6 \pm 3.3$ & $<0.001^{\mathrm{a}}$ \\
\hline LVEDV (mL) & $75.7 \pm 19.9$ & $85.9 \pm 26.2$ & $71.6 \pm 15.5$ & 0.04 \\
\hline LVESV (mL) & $31.2 \pm 11.5$ & $36.6 \pm 15.5$ & $28.9 \pm 8.8$ & 0.06 \\
\hline LAVI $\left(\mathrm{mL} / \mathrm{m}^{2}\right)$ & $21.9 \pm 7.4$ & $25.4 \pm 10.3$ & $20.5 \pm 5.5$ & 0.06 \\
\hline $\operatorname{LVM}(\mathrm{g})$ & $152.2 \pm 42.1$ & $192.9 \pm 38.8$ & $135.5 \pm 30.8$ & $<0.001^{\mathrm{a}}$ \\
\hline $\operatorname{LVMI}\left(\mathrm{g} / \mathrm{m}^{2}\right)$ & $78.9 \pm 20.4$ & $99.7 \pm 19.3$ & $70.5 \pm 13.8$ & $<0.001^{\mathrm{a}}$ \\
\hline $\mathrm{EF}(\%)$ & $58.7(55-63.3)$ & $55(50-64)$ & $59(56-63)$ & 0.11 \\
\hline LVGLS (-\%) & $-19.5(-21.6$ to -17.6$)$ & $-19.4(-20.3$ to -17$)$ & $-19.5(-21.7$ to -17.5$)$ & 0.19 \\
\hline Patients with GLS $\geq-18 \%$ & $11(28.9 \%)$ & $4(36.4 \%)$ & $7(25.9 \%)$ & 0.52 \\
\hline \multicolumn{5}{|l|}{ Transmitral flow parameters } \\
\hline E wave velocity $(\mathrm{cm} / \mathrm{s})$ & $70.8 \pm 18.5$ & $68.8 \pm 22.8$ & $71.6 \pm 16.8$ & 0.67 \\
\hline A wave velocity $(\mathrm{cm} / \mathrm{s})$ & $78.9 \pm 21.4$ & $85.7 \pm 19.5$ & $76.2 \pm 21.8$ & 0.22 \\
\hline E/A ratio (medial) & $0.96 \pm 0.35$ & $0.82 \pm 0.3$ & $1 \pm 0.4$ & 0.14 \\
\hline E wave deceleration time(s) & $225.3 \pm 61.1$ & $214.3 \pm 58.6$ & $229.8 \pm 62.7$ & 0.49 \\
\hline \multicolumn{5}{|l|}{ Tissue Doppler parameters } \\
\hline Septal e' $(\mathrm{cm} / \mathrm{s})$ & $8.1 \pm 2.7$ & $6.7 \pm 2.3$ & $8.7 \pm 2.7$ & 0.04 \\
\hline Lateral e' (cm/s) & $10.8 \pm 3.5$ & $10.1 \pm 3.4$ & $11.1 \pm 3.5$ & 0.42 \\
\hline E/mean e' & $7.9 \pm 2.6$ & $8.5 \pm 2.7$ & $7.8 \pm 2.6$ & 0.41 \\
\hline $\mathrm{RV}(\mathrm{mm})$ & $34 \pm 3.8$ & $34.6 \pm 4.3$ & $33.7 \pm 3.5$ & 0.49 \\
\hline TAPSE (mm) & $23 \pm 3.6$ & $23 \pm 4.1$ & $23 \pm 3.5$ & 0.99 \\
\hline RV S'(cm/s) & $16.5 \pm 2.8$ & $16.7 \pm 3.4$ & $16.3 \pm 2.3$ & 0.54 \\
\hline PAP systolic (mmHg) & $23.4 \pm 5.1$ & $23.7 \pm 4.8$ & $23.2 \pm 5.3$ & 0.82 \\
\hline
\end{tabular}

$h s-T n I$ high sensitive troponin-I, $L V D d$ left ventricular end diastolic diameter, IVSd interventricular septum diameter, $P W d$ posterior wall diameter, $L A d$ left atrium diameter, $L V E D V$ left ventricular end diastolic volume, $L V E S V$ left ventricular end systolic volume, $L A V I$ left atrium volume index, $L V M$ left ventricular mass, $L V M I$ left ventricular mass index, $E F$ ejection fraction, $L V G L S$ left ventricular global longitudinal strain, $P A P$ pulmonary artery pressure, $R V$ right ventricle, TAPSE tricuspid annular plane systolic excursion, $S$ ' tricuspid lateral annular systolic velocity

${ }^{a}$ Mann Whitney-U test

the two groups. Baseline echocardiographic parameters and clinical endpoints of the study population according to age were described in Table 3. LV end diastolic and left atrial diameters, thickness of the interventricular septum, LVM, LVMI and need for high flow oxygen tended to be higher in the age $\geq 65$ group. However, EF and septal e' wave velocity were significantly lower in the elderly group. LVGLS values, number of patients with LVGLS $>-18$, admission to ICU and need for intubation tended to be higher in the elderly group but these findings did not reach statistically significance. Other echocardiographic parameters and clinical endpoints showed no difference between the groups.

LVGLS according to the troponin levels and in-hospital adverse outcomes were shown at Fig. $3 a$ and $b$. There was no statistically significant difference in terms of LVGLS according to the troponin levels and in-hospital adverse outcomes. We performed an additional multivariate logistic regression analysis for the predictors of the LVGLS $\geq-18 \%$ among variables such as age, hs-TnI, CRP, glucose, procalcitonin and HTN. Among these variables only glucose level was found as an independent predictor of LVGLS $\geq-18 \%$ [Odds ratio(OR) 1.04, 95\% Confidence Interval(CI) 1.004-1.08, p 0.03].

The incidence of single and combined adverse outcomes of the study groups were depicted in Fig. 4a and b. Highflow oxygen and invasive mechanical ventilation therapy demand and the number of ICU admissions were noticeably more frequent in the high troponin group. We performed a multivariate logistic regression analysis to evaluate the independent predictors of combined in hospital clinical adverse events among these variables such as age, hs-TnI, CRP, procalcitonin and lymphocyte count. Hs-TnI, CRP and procalcitonin were found as independent predictors of in hospital clinical adverse events (OR 1.11, 95\% CI 1.06-1.16, p 0.05 for hs-TnI; OR 1.05, 95\% CI 1.007-1.09, p 0.02 for CRP; OR 1.04, 95\% CI 1.001-1.06, p 0.03 for procalcitonin) We also performed Hosmer-Lemeshow goodness of fit test for our model and $\mathrm{p}$ value was found as 0.43 . Nagelkerke $\mathrm{R}$ 
Table 3 The echocardiographic variables and clinical endpoints of the study group according to age

\begin{tabular}{|c|c|c|c|c|}
\hline & All patients $(n=38)$ & Age $\geq 65$ years $(n=11)$ & Age $<65$ years $(n=27)$ & $\mathrm{p}$ value \\
\hline LVDd (mm) & $44.3 \pm 4.1$ & $46.3 \pm 5.1$ & $43.5 \pm 3.4$ & 0.05 \\
\hline IVSd (mm) & $10.1 \pm 1.5$ & $10.9 \pm 1.6$ & $9.8 \pm 1.3$ & $0.05^{\mathrm{a}}$ \\
\hline PWd (mm) & $9.9 \pm 1.3$ & $10.6 \pm 1.3$ & $9.7 \pm 1.2$ & $0.06^{\mathrm{a}}$ \\
\hline LAd (mm) & $35.6 \pm 4.7$ & $39.8 \pm 4.8$ & $33.8 \pm 3.4$ & $0.001^{\mathrm{a}}$ \\
\hline LVEDV (mL) & $81.1 \pm 27.5$ & $85.9 \pm 26.2$ & $73.5 \pm 15.9$ & 0.29 \\
\hline LVESV (mL) & $31.2 \pm 11.5$ & $35.9 \pm 16$ & $29.2 \pm 8.6$ & 0.21 \\
\hline LAVI $\left(\mathrm{mL} / \mathrm{m}^{2}\right)$ & $21.9 \pm 7.4$ & $22.3 \pm 10.9$ & $21.8 \pm 5.6$ & 0.86 \\
\hline $\operatorname{LVM}(\mathrm{g})$ & $152.2 \pm 42.1$ & $180.3 \pm 51.6$ & $140.7 \pm 31.9$ & $0.02^{\mathrm{a}}$ \\
\hline $\operatorname{LVMI}\left(\mathrm{g} / \mathrm{m}^{2}\right)$ & $78.9 \pm 20.4$ & $95.5 \pm 24.1$ & $72.2 \pm 14.4$ & $0.004^{\mathrm{a}}$ \\
\hline $\mathrm{EF}(\%)$ & $58.7(55-63.3)$ & $56(43-64)$ & $59(56-63)$ & 0.02 \\
\hline LVGLS (-\%) & $-19.5(-21.6$ to -17.6$)$ & $-18.3(-21.6$ to -11.6$)$ & $-19.9(-21.7$ to -18.2$)$ & 0.09 \\
\hline Patients with GLS $\geq-18 \%$ & $11(28.9 \%)$ & $5(45.5 \%)$ & $6(22.2 \%)$ & 0.15 \\
\hline \multicolumn{5}{|l|}{ Transmitral flow parameters } \\
\hline E wave velocity $(\mathrm{cm} / \mathrm{s})$ & $70.8 \pm 18.5$ & $73.6 \pm 22.6$ & $69.7 \pm 16.9$ & 0.55 \\
\hline A wave velocity $(\mathrm{cm} / \mathrm{s})$ & $78.9 \pm 21.4$ & $83.8 \pm 12.5$ & $76.9 \pm 24$ & 0.37 \\
\hline E/A ratio (medial) & $0.96 \pm 0.35$ & $0.89 \pm 0.3$ & $0.98 \pm 0.4$ & 0.51 \\
\hline E wave deceleration time(s) & $225.3 \pm 61.1$ & $216.1 \pm 54.5$ & $229.1 \pm 64.2$ & 0.56 \\
\hline \multicolumn{5}{|l|}{ Tissue Doppler parameters } \\
\hline Septal e' $(\mathrm{cm} / \mathrm{s})$ & $8.1 \pm 2.7$ & $6.8 \pm 2.1$ & $8.6 \pm 2.8$ & 0.03 \\
\hline Lateral e' $(\mathrm{cm} / \mathrm{s})$ & $10.8 \pm 3.5$ & $9.7 \pm 3.3$ & $11.3 \pm 3.5$ & 0.22 \\
\hline E/mean e' & $7.9 \pm 2.6$ & $9.2 \pm 2.7$ & $7.5 \pm 2.5$ & 0.06 \\
\hline $\mathrm{RV}(\mathrm{mm})$ & $34 \pm 3.8$ & $34.8 \pm 4.5$ & $33.6 \pm 3.4$ & 0.38 \\
\hline TAPSE (mm) & $23 \pm 3.6$ & $23 \pm 4.2$ & $23 \pm 3.4$ & 0.98 \\
\hline RV S'(cm/s) & $16.5 \pm 2.8$ & $16.1 \pm 3.8$ & $14.3 \pm 2.3$ & 0.09 \\
\hline PAP systolic (mmHg) & $23.4 \pm 5.1$ & $23.6 \pm 4.9$ & $23.3 \pm 5.6$ & 0.89 \\
\hline \multicolumn{5}{|l|}{ Clinical endpoints } \\
\hline ICU admission & $7(18.4 \%)$ & $4(36.4 \%)$ & $3(11.1 \%)$ & 0.07 \\
\hline High flow oxygen & $13(34.2 \%)$ & $7(63.6 \%)$ & $6(22.2 \%)$ & 0.02 \\
\hline Intubation & $3(7.9 \%)$ & $1(9.1 \%)$ & $2(7.4 \%)$ & 0.86 \\
\hline
\end{tabular}

$L V D d$ left ventricular end diastolic diameter, IVSd interventicular septum diameter, $P W d$ posterior wall diameter, $L A d$ left atrium diameter, $L V E D V$ left ventricular end diastolic volume, $L V E S V$ left ventricular end systolic volume, $L A V I$ left atrium volume index, $L V M$ left ventricular mass, $L V M I$ left ventricular mass index, $E F$ ejection fraction, $L V G L S$ left ventricular global longitudinal strain, $P A P$ pulmonary artery pressure, $R V$ right ventricle, TAPSE tricuspid annular plane systolic excursion, $S$ ' tricuspid lateral annular systolic velocity

${ }^{a}$ Mann Whitney-U test

square value was found as 0.78 . These tests demonstrated that calibration of our model to predict in hospital adverse events was accurate in our study.

\section{Discussion}

This study demonstrated no statistically significant difference in LVGLS between positive troponin and negative troponin groups in patients with COVID-19 pneumonia. Additionally, our study showed that LV systolic function parameters slightly decreased in positive troponin group compared to negative troponin group; but both were still within normal limits. Similar to the literature, troponin level was found to be an independent predictor of adverse clinical outcomes which were significantly more common in the positive troponin group [11].

Many studies have previously reported the unfavourable effect of DM [12, 13] and HTN [14] on LV mechanics. In our study glucose level was found as an independent predictor of LVGLS $\geq-18 \%$ as compatible with the literature. A study conducted by Azizi MS et al. showed a progressive decline in both EF and LVGLS measurements with increasing age, thereby resulting in increased frequency of morbidity and mortality [15]. Similarly, we found that elderly patients had worse LVGLS/EF levels and had more unfavourable clinical outcomes. In most of the reports concerning COVID-19, older age, male gender, presence of HTN, DM or cardiovascular disease had been identified as the cardinal risk factors predicting mortality. Thus, no death was encountered in our 

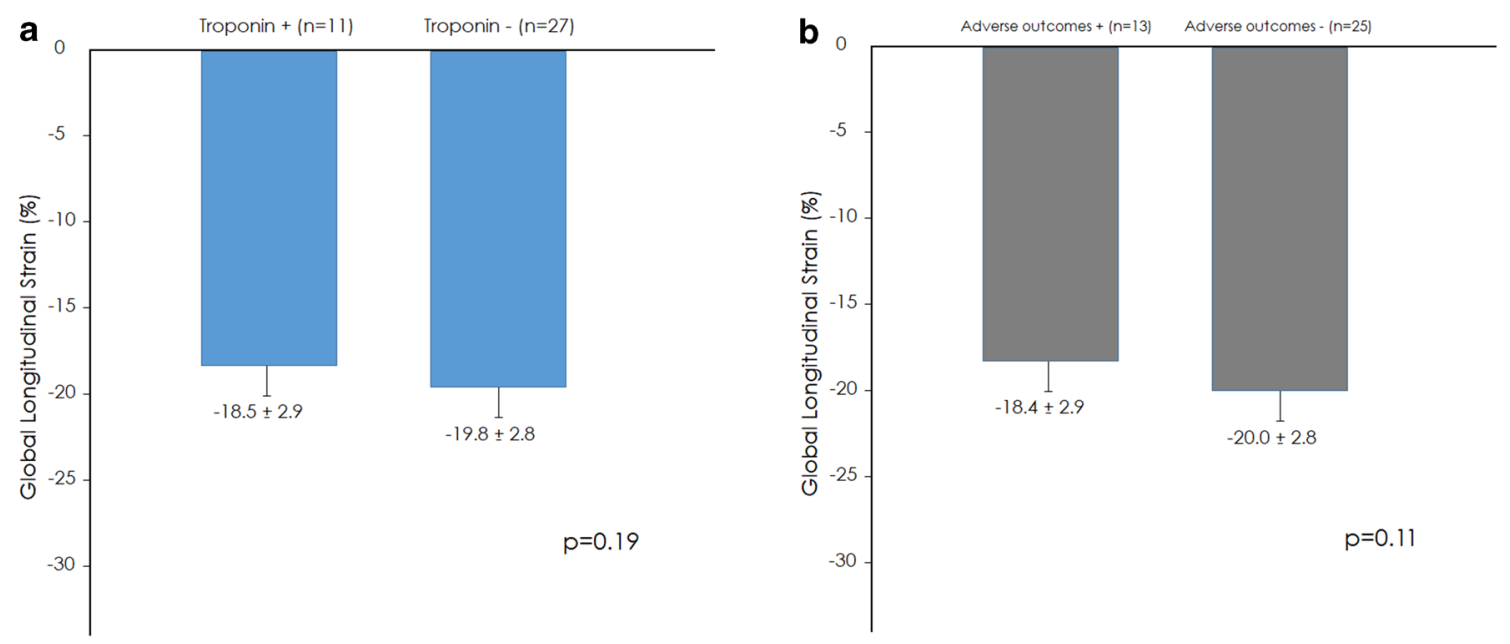

Fig. 3 a Global longitudinal strain values of the study group according to Hs-TnI levels. b Global longitudinal strain levels according to presence of adverse outcomes. Data are presented as mean \pm SD
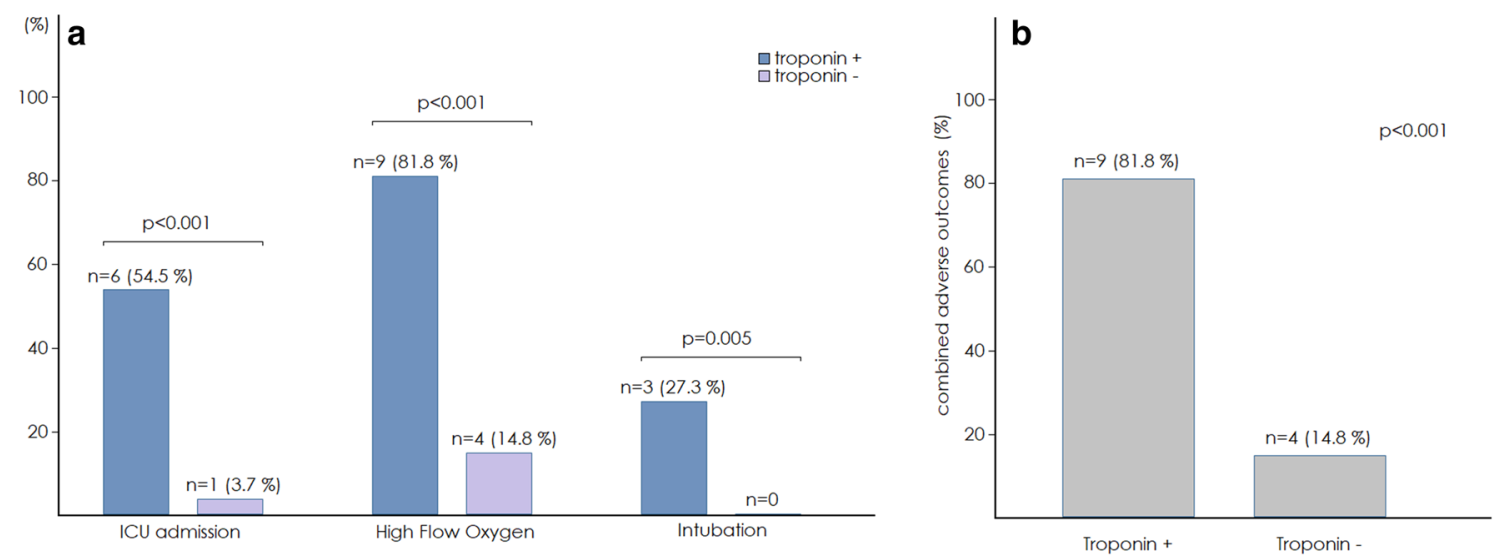

Fig. 4 a The incidence of single in hospital adverse outcomes of the study groups according to Hs-TnI levels, $\mathbf{b}$ The ratio of combined adverse outcomes in patient groups according to Hs-TnI levels

study during the in-hospital period which may be owing to the exclusion of patients with these high risk factors during recruitment.

Comorbidities like advanced age, uncontrolled HTN or DM are also known to influence coronary microcirculation via increasing intracellular calcium and calcium sensitization in vascular smooth muscle cells, thereby causing coronary constriction and ischemia $[16,17]$. It is not surprising to detect an increase in serum hs-TnI levels and a reduction in quantitative LV systolic function parameters in this patient group. Therefore, patients with these severe comorbidities were not enrolled in our study group to evaluate the exact effect of troponin increase caused by COVID-19 penumonia itself on both LV mechanics and adverse clinical outcomes. Although we excluded such patients, the positive troponin group was still older and had a slightly higher incidence of HTN. A mild decrease in both EF and GLS measurements in this group of patients might be explained by the same pathophysiology mentioned above. In other words, a decrease in LVGLS measurement might be due to older age and higher incidence of HTN of the patients in the positive troponin group rather than an elevation of the troponin levels.

Echocardiographic parameters related to the left atrium and left ventricle geometry and left ventricular mass did not demonstrate any significant change between the two groups. Compared with the negative troponin group; the left atrium and left ventricle were more dilated, interventricular septum, LV posterior wall thickness, LV mass and mass indexes were more increased in the positive troponin group. This may also be explained by a relatively high incidence of HTN and older age in the positive troponin group. The available evidence in this study does not suggest a major difference in RV diameter, RV systolic function parameters (TAPSE, RV S') and LV diastolic function measurements 
between the two groups. On the contrary, a recent study performed by Lie Y, et al. [18] demonstrated a statistically significant association between RV longitudinal strain and mortality in patients with COVID-19. RV strain was also detected as an independent predictor of mortality. Most notably, whole patients diagnosed with COVID-19 were included in that study without categorizing them according to their comorbid status. Furthermore, detection of RV longitudinal strain as an independent predictor of mortality might be due to the enrollment of a relatively high number of patients with significant comorbidities.

Even though LV systolic function parameters mildly decreased in positive troponin group, its direct effect on morbidity and mortality was not detected in our results. This may be associated with the poor course of the infection and detection of prominently high levels of parameters related to the severity of disease like ferritin, $\mathrm{d}$ dimer or $\mathrm{LDH}$ and acute phase reactants in the positive troponin group. Higher incidence of in hospital adverse clinical outcomes may be related to the worse course of the disease instead of slight decrease in systolic function measurements of the positive troponin group. Our findings were also condordant with this point of view, as CRP and procalcitonin levels were found as independent predictors of adverse clinical events in our study group. According to a metaanalysis that consisted of patients with sepsis, as the infection became more severe, LVGLS further decreased and lower GLS values were associated with higher mortality [19]. Although we have a different study population, increased systemic inflammatory response in the positive troponin group may contribute to subtle LV dysfunction in COVID-19 patients. Meanwhile, it is worth noting that the troponin increase may be considered as a sign of in-hospital adverse clinical outcomes in this group of patients. Thus, closer attention and follow up should be engaged in to improve adverse clinical outcomes for this group.

There were some limitations to be noted in our study. The lack of significant decline in LVGLS values in the positive troponin group may be related to our relatively small sample size since these results were based on a single center study. Since we aimed to investigate the relation between troponin increase caused by pneumonia itself and LVGLS, we had to exclude patients with severe comorbidities which may impair strain analysis. For this reason, we had to enroll a small group of all hospitalized COVID patients to analyze a homogeneous population. Also small sample size of our study group may limit to establish any causal relationship between troponin increase and LV mechanics in patients with COVID-19 pneumonia.

In conclusion, our results showed that troponin increase is highly related to in-hospital adverse events; however no relationship was found between troponin increase and LVGLS values of COVID-19 patients. Nevertheless, further studies are needed to elucidate the association between LV systolic function parameters and troponin levels to confirm our findings in a larger cohort of patients.

Acknowledgements English grammar editing of the study was performed by Editage.

Funding None.

\section{Compliance with ethical standards}

Conflict of interest All the authors declared that they have no conflict of interest.

\section{References}

1. Huang C, Wang Y, Li X et al (2020) Clinical features of patients infected with 2019 novel coronavirus in Wuhan, China. Lancet 395:497-506

2. Wang D, Hu B, Hu C et al (2020) Clinical characteristics of 138 hospitalized patients with 2019 novel coronavirus-1nfected pneumonia in Wuhan China. JAMA 323:1061-1069

3. Deng Q, Hu B, Zhang Y et al (2020) Suspected myocardial injury in patients with COVID-19: evidence from front-line clinical observation in Wuhan, China. Int J Cardiol. https://doi. org/10.1016/j.ijcard.2020.03.087

4. Guo T, Fan Y, Chen M et al (2020) Cardiovascular implications of fatal outcomes of patients with coronavirus disease (COVID-19). JAMA Cardiol. https://doi.org/10.1001/jamacardio.2020.1017

5. Zhou B, She J, Wang Y et al (2020) The clinical characteristics of myocardial injury in severe and very severe patients with novel coronavirus disease. J Infect. https://doi.org/10.1016/j. jinf.2020.03.021

6. Potter E, Marwick TH (2018) Assessment of left ventricular function by echocardiography: the case for routinely adding global longitudinal strain to ejection fraction. JACC Cardiovasc Imaging 11:260-274

7. Jung IH, Park JH, Lee JA et al (2020) Left ventricular global longitudinal strain as a predictor for left ventricular reverse remodeling in dilated cardiomyopathy. J Cardiovasc Imaging 28:137-149

8. Lyngbakken MN, Aagaard EN, Kvisvik B et al (2020) Cardiac troponin I and T are associated with left ventricular function and structure: data from the akershus cardiac examination 1950 study. Clin Chem 66:567-578

9. Lang RM, Badano LP, Mor-Avi V et al (2015) Recommendations for cardiac chamber quantification by echocardiography in adults: an update from the American Society of Echocardiography and the European Association of Cardiovascular Imaging. J Am Soc Echocardiogr 28:1-39

10. Marwick TH, Leano RL, Brown J et al (2009) Myocardial strain measurement with 2-dimensional speckle-tracking echocardiography: definition of normal range. JACC Cardiovasc Imaging 2:80-84

11. Shi S, Qin M, Shen B et al (2020) Association of cardiac injury with mortality in hospitalized patients with COVID-19 in Wuhan, China. JAMA Cardiol. https://doi.org/10.1001/jamacardio .2020 .0950

12. Roos CJ, Scholte AJ, Kharagjitsingh AV et al (2014) Changes in multidirectional LV strain in asymptomatic patients with type 2 diabetes mellitus: a 2-year follow-up study. Eur Heart J Cardiovasc Imaging 15:41-47 
13. Zoroufian A, Razmi T, Taghavi-Shavazi M et al (2014) Evaluation of subclinical left ventricular dysfunction in diabetic patients: longitudinal strain velocities and left ventricular dyssynchrony by two-dimensional speckle tracking echocardiography study. Echocardiography 31:456-463

14. Mu Y, Qin C, Wang C et al (2010) Two-dimensional ultra-sound speckle tracking imaging in evaluation of early changes in left ventricular diastolic function in patients with essential hypertension. Echocardiography 27:146-154

15. Azizi MS, Nasution SA, Setiati S et al (2020) Global longitudinal strain (GLS) in elderly and its associated factors. Acta Med Indones 52:47-54

16. Feihl F, Liaudet L, Waeber B (2009) The macrocirculation and microcirculation of hypertension. Curr Hypertens Rep 11(3):182-189

17. Jia G, DeMarco VG, Sowers JR (2016) Insulin resistance and hyper-insulinaemia in diabetic cardiomyopathy. Nat Rev Endocrinol 12:144-153
18. Li Y, Li Hi Zhu S, Xie Y et al (2020) Prognostic value of right ventricular longitudinal strain in patients with COVID-19. JACC Cardiovasc Imaging. https://doi.org/10.1016/j.jcmg.2020.04.014

19. Sanfilippo F, Corredor C, Fletcher N et al (2018) Left ventricular systolic function evaluated by strain echocardiography and relationship with mortality in patients with severe sepsis or septic shock: a systematic review and meta-analysis. Crit Care 22:183

Publisher's Note Springer Nature remains neutral with regard to jurisdictional claims in published maps and institutional affiliations. 